\title{
Wo findet sich Erfahrung im Hirn?
}

\section{Cord Benecke}

Online publiziert: 1. Februar 2012

(C) Springer-Verlag 2012

Die Beiträge zum Schwerpunkt „Psychoanalyse und Neurowissenschaften“ können vielleicht am ehesten durch die oben gestellte Frage verbunden werden. Es ist in den letzten Jahren ja viel geschrieben worden zu möglichen und unmöglichen Verbindungen zwischen Psychoanalyse und Neurowissenschaften - von euphorischen Begeisterungsstürmen über die Nachweise Freud'scher Kernpositionen (wie zum Beispiel die Dominanz unbewusster Prozesse) bis zum Absprechen jeglicher Relevanz der Hirnforschung für die Psychoanalyse.

Die drei Beiträge versuchen diese Polarisierungen im Wesentlichen zu vermeiden, woraus sich zwangsläufig eher viele offene Fragen ergeben und wenig klare Antworten. In diesem Sinne können die Beiträge vielleicht als Anregung zum Nachdenken über die und als Aufruf zum Beforschen der diskutierten Themen verstanden werden. Da gibt es noch viel zu tun.

Die Arbeit von Rainer Krause über die Psychodynamik der Gedächtnisfunktion gibt einen Überblick über die weder von (neuro-)psychologischer noch von psychoanalytischer Seite bisher befriedigend gelösten Probleme der Funktionsweise des Gedächtnisses. Man sollte eigentlich meinen, dass das Gedächtnis als ein für jede Facette psychoanalytischer Theorie so zentraler Gegenstands- bzw. Funktionsbereich, differenziert ausgearbeitet und theoretisch sowie klinisch durchdrungen wäre. Dass dem nicht so ist, zeigt die Arbeit von Krause: Im Gegenteil wird deutlich, dass wir von einem Gedächtnismodell, das die unterschiedlichen Phänomene des Speicherns, des Erinnerns und der Amnesien, der Umwandlungen, Überformungen und Neukonstruktionen von Gedächtnisinhalten bei unterschiedlichen psychischen Störungen und innerhalb von psychotherapeutischen Veränderungsprozessen etc. auch nur halbwegs kohärent integrieren könnte, weit entfernt sind.

Prof. Dr. phil. C. Benecke $(\bowtie)$

Institut für Psychologie, Universität Kassel,

Holländische Straße 36-38, 34127 Kassel, Deutschland

E-Mail: benecke@uni-kassel.de 
Ebenso ist die Frage von Jorge Canestri, wie eigentlich Sprache als das Mittel der Psychotherapie und Psychoanalyse zur „Förderung der psychischen Veränderung des Patienten“ beiträgt, mitnichten geklärt. Die Fundierung der Funktion der Sprache ist für eine Veränderungstheorie keineswegs trivial, scheint aber nach Lektüre von Canestri eher ein noch recht offenes Forschungsfeld zu sein.

Der Beitrag von Matthias Richter über Sinn und Unsinn einer Neuropsychotherapie geht das Verhältnis der Disziplinen Neurowissenschaften und Psychotherapie nochmal ganz grundsätzlich an, indem er im Wesentlichen auf die prinzipiellen Unterschiede zwischen Betrachtungs- und Forschungsebenen hinweist (zum Beispiel die psychophysiologischen Zustände im Unterschied zu subjektiven Erfahrungen). Der Beitrag wird im nächsten Heft von Hans-Peter Kapfhammer differenziert kommentiert und reflektiert. Ich hoffe, dass das vorliegende Heft des Forum der Psychoanalyse insgesamt zu einer gewinnbringenden und anregenden Lektüre wird.

Cord Benecke, Prof. Dr. phil. Dipl.-Psych., Jg. 1965; psychologischer Psychotherapeut, Psychoanalytiker. Professur für klinische Psychologie und Psychotherapie am Institut für Psychologie der Universität Kassel. Leiter der Forschungskommission der Deutschen Psychoanalytischen Gesellschaft. Forschungsschwerpunkte: klinische Emotionsforschung; Psychotherapieforschung. Mitglied des Herausgebergremiums dieser Zeitschrift. 